\title{
Cloud Computing Virtualization
}

\author{
Mohd Saleem \\ Assistant Professor \\ Soet, Baba Ghulam Shah Badshah University \\ Rajouri, J\&K
}

\begin{abstract}
Cloud computing is the currently biggest technology in this environment that changes the thinking of the whole world. The main aim of the cloud computing is to provide services on line depending upon the need of the users of the cloud. Cloud uses internet to provide its services with main focus on cost reduction, hardware reduction and pay only for the services that user want to use. Virtualization play a big role in cloud computing. Virtualization is a technique like cost saving, hardware reducing and energy saving used by the cloud provider. In this paper we discussed in detail about virtualization and its different types.
\end{abstract}

Keywords: Virtualization; Virtualization Architecture; Hypervisor; Types of hypervisor; Virtualization technologies

\section{INTRODUCTION}

Cloud computing is a technology that provides online services to the user on demand that reduces various software and hardware maintenances for individual level. Virtualization allows multiple instances of an operating to run concurrently on single computer. It is an abstraction over physical resources to make them shareable among multiple physical users. For resources to be shareable by number of physical user it allocates a logical name to a physical resource and enables a pointer to that physical resource on demand.

\section{VIRTUALIZATION ARCHITECTURE}

Virtual machine is computer software that runs operating system and applications. It is the duplication of real machine. The physical server on which one or more virtual machines are running is defined as host. The virtual machines are called guests. Multiple virtual systems (VMs) can run on a single physical system. This is shown in figure 1 .

\begin{tabular}{|c|c|c|c|}
\hline \multirow[t]{2}{*}{ Applications } & \multirow[t]{2}{*}{ Applications } & \multirow[b]{3}{*}{$\bullet \bullet \bullet$} & \\
\hline & & & Applications \\
\hline $\begin{array}{l}\text { Virtual } \\
\text { Machine } \\
\text { (VM) }\end{array}$ & $\begin{array}{l}\text { Virtual } \\
\text { Machine } \\
\text { (VM) }\end{array}$ & & $\begin{array}{c}\text { Virtual Machine } \\
\text { (VM) }\end{array}$ \\
\hline \multicolumn{4}{|c|}{ Virtualization Platform } \\
\hline \multicolumn{4}{|c|}{ Physical Resources } \\
\hline
\end{tabular}

Figure.1 Sample Architecture

\section{HYPERVISOR}

It is a program that allows multiple operating systems to share a single hardware host. Actually it a low-level program that provide system resource access to the virtual machines.

\subsection{Type-1 hypervisor}

It is also called as native or bare metal. This type of hypervisor runs directly on host hardware to manage guest
Operating system. It does not depend upon the operating system. This hypervisor support hardware virtualization. This is shown in figure 2 .

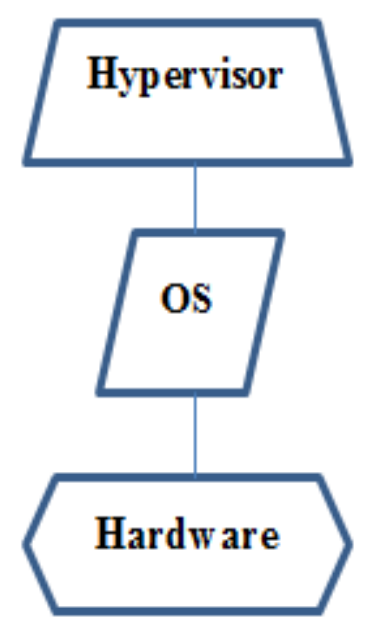

Figure.2 Type1 Hypervisor

\subsection{Type 2 hypervisor}

It is a type of client hypervisor that sits on top of an operating system. It cannot run until the operating system is already running. Software virtualization is carried out in this hypervisor because it depends upon operating system. If the operating system fails then all end users are affected as shown in figure 3 .

These hypervisors are basically like applications that install on a guest OS. Containers, KVM, Microsoft Hyper V, VMware Fusion, Virtual Server 2005 R2, Windows Virtual $\mathrm{PC}$ and VMware workstation 6.0 come under the category of this hypervisor. Software virtualization provides better hardware compatibility than bare-metal virtualization, because the OS is responsible for the hardware drivers instead of the hypervisor. 


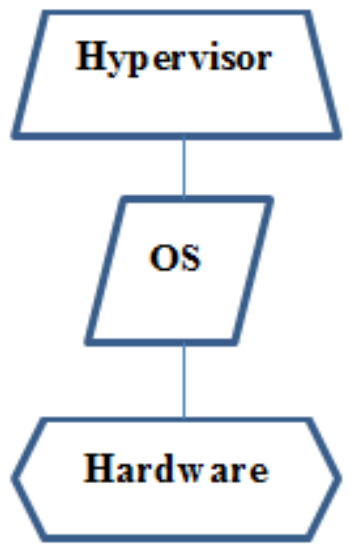

Figure.3 Type-2 hypervisor

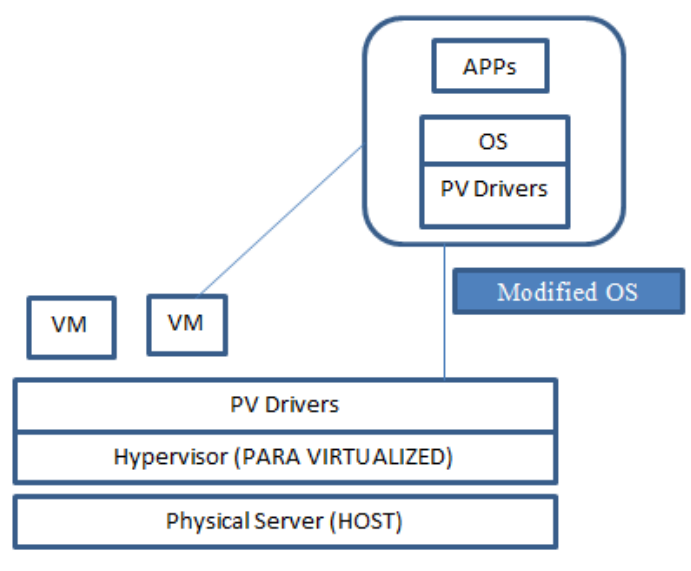

Figure.5 Para Virtualization

\section{VIRTUALIZATION TECHNOLOGIES}

\subsection{Full Virtualization}

Full virtualization is a technique in which a complete installation of one machine is run on another. This virtualization support different operating system but it requires specific hardware combination. The hypervisor interacts directly with the physical server's CPU and disk space as shown in figure 4 . In this virtualization each virtual server is completely unaware of other virtual servers that are currently running on the physical machine.

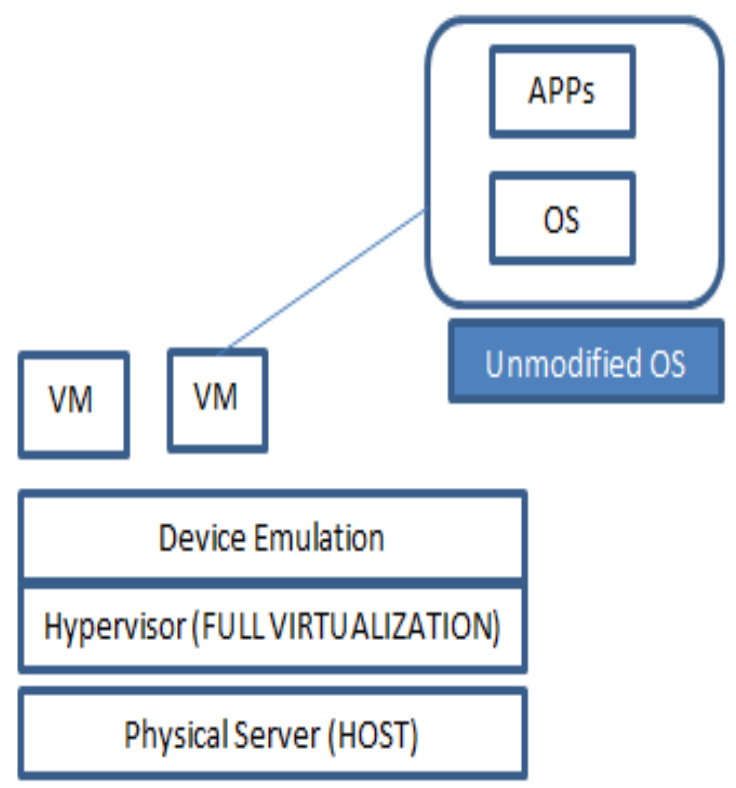

Figure.4 Full Virtualization

\subsection{Para Virtualization}

Here the guest operating system is aware that it's being virtualized. Due to this advance information the guest operating system can short circuit its drivers to minimize the overhead of communicating with physical devices. This virtualization removes the drawback of full virtualization. This is shown in figure 5.

\subsection{OS Level Virtualization}

This technique does not use any hypervisor. It is responsibility of the host OS to performs all the functions of a fully virtualized hypervisor as shown in figure 6 . The guest servers must run the same OS due to this it is called homogeneous environment.

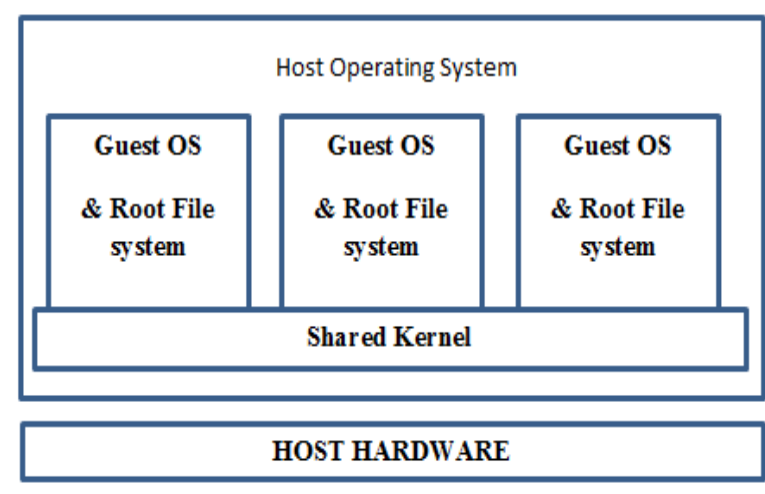

Figure.6 OS level Virtualization

\subsection{Hardware-assisted Virtualization}

This approach enables efficient full virtualization using help from hardware capabilities, primarily from the host processors. It is also known as accelerated virtualization and it was added to x86 processors in 2006. The Server hardware is virtualization aware. In this type the hypervisor and VMM load at privilege Ring -1(Firmware) as shown in fig 6 .

Hardware-assisted virtualization reduces the maintenance overhead of Para-virtualization as it reduces the changes needed in the guest operating system. Better performance can be obtained by using this virtualization.

A pure hardware-assisted virtualization approach, using unmodified guest operating systems, involves many VM traps, and thus high CPU overheads, limiting scalability and the efficiency of server consolidation. This performance hit can be mitigated by the use of Para virtualized drivers; the combination has been called hybrid virtualization. Hardware-assisted virtualization requires explicit support in 
the host CPU, which is not available on all -x86/x86_64 processors.

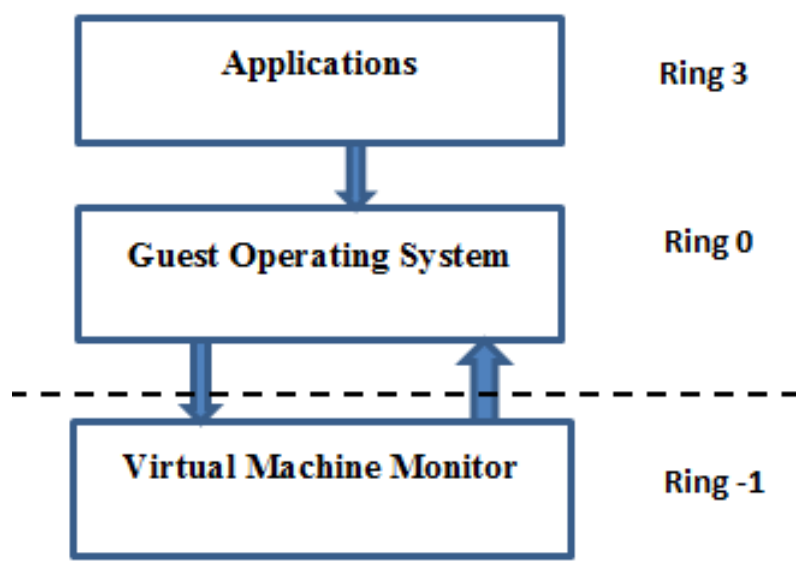

Figure.7 Hardware-assisted Virtualization

\section{CONCLUSION}

Cloud computing is widely used in large enterprise for providing services to the user depending upon the need and user have to pay for the services that it used. Virtualization is a technique in cloud computing that help in saving the hardware implementation cost for different operating system because it allow sharing of single physical system resources. In this paper we discussed in detail about virtualization and its different types.

\section{REFERENCES}

[1] O. Agesen, A. Garthwaite, J. Sheldon, and P. Subrahmanyam. The evolution of an x86 virtual machine monitor. ACM SIGOPS Operating Systems Review, 44(4):3-18, 2010. .

[2] Secure virtualization for cloud computing".Flavio Lombardi, Roberto Di Pietro, June 2010.

[3] Shyam Patidar; Dheeraj Rane; Pritesh Jain "A Survey Paper on Cloud Computing" in proceeding of Second International Conference on Advanced Computing \& Communication Technologies, 2012

[4] T. Dillon, C. Wu, and E. Chang, "Cloud Computing: Issues and Challenges," 2010 24th IEEE International Conference on Advanced Information Networking and Applications(AINA), pp. 27-33, DOI= 20-23 April 2010.

[5] Sannella, M. J. 1994 Constraint Satisfaction and Debugging for Interactive User Interfaces. Doctoral Thesis. UMI Order Number: UMI Order No. GAX9509398., University of Washington.

[6] Forman, G. 2003. An extensive empirical study of feature selection metrics for text classification. J. Mach. Learn. Res. 3 (Mar. 2003), 1289-1305.

[7] Brown, L. D., Hua, H., and Gao, C. 2003. A widget framework for augmented interaction in SCAPE.
[8] Y.T. Yu, M.F. Lau, "A comparison of MC/DC, MUMCUT and several other coverage criteria for logical decisions", Journal of Systems and Software, 2005, in press.

[9] Spector, A. Z. 1989. Achieving application requirements. In Distributed Systems, S. Mullender. 